\title{
Increased Relaxant Action of Forskolin and Isoproterenol against Muscarinic Agonist-Induced Contractions in Smooth Muscle from $\mathrm{M}_{2}$ Receptor Knockout Mice ${ }^{\mathrm{a}}$
}

Minoru Matsui, ${ }^{1,2}$ Michael T. Griffin, ${ }^{3}$ Darakhshanda Shehnaz, Makoto M. Taketo ${ }^{2,6}$ and Frederick J. Ehlert ${ }^{5}$

${ }^{1}$ Division of Neuronal Network, Department of Basic Medical Sciences, The Institute of Medical Science, The University of Tokyo, Minato-ku, Tokyo 108-8639, Japan.

2 Laboratory of Biomedical Genetics, Graduate School of Pharmaceutical Sciences, The University of Tokyo, Bunkyo-ku, Tokyo 113-0033, Japan.

3 Department of Environmental and Chemical Sciences, Chapman University, Orange, California 92866.

${ }^{4}$ Banting and Best Department of Medical Research, University of Toronto, Ontario, Canada M5G1L6.

5 Department of Pharmacology, College of Medicine, University of California, Irvine, Irvine, California 92697-4625.

${ }^{6}$ Department of Pharmacology, Graduate School of Medicine, Kyoto University, Sakyo-ku, Kyoto 606-8501.

1,4,6 Present address 
Running title: $\mathrm{M}_{2}$ muscarinic receptor knockout mice and smooth muscle

To whom correspondence should be addressed:

Frederick J. Ehlert, Ph.D.

Department of Pharmacology

University of California, Irvine

Irvine, California 92697-4625

Tel: 949-824-3709

FAX: 949-824-4855

e-mail: fjehlert@uci.edu

Number of text pages: 15

Number of tables: 4

Number of figures: 3

Number of references: 37

Number of words in Abstract: 238

Number of words in Introduction: 572

Number of words in Discussion: 1,612

Abbreviations: $\quad E C_{50}$, concentration of agonist eliciting half-maximal response; KO, knockout; KRB, Krebs Ringer Bicarbonate; $E_{\max }$, maximal response.

Section assignment: Gastrointestinal, Hepatic, Pulmonary and Renal 
The ability of forskolin and isoproterenol to inhibit the contractile action of the muscarinic agonist, oxotremorine-M, was investigated in smooth muscle from wild type and $\mathrm{M}_{2}$ muscarinic receptor knockout mice. Forskolin $(5.0 \mu \mathrm{M})$ caused a significant reduction in the contractile activity of oxotremorine-M in ileum, trachea and urinary bladder from both wild type and $\mathrm{M}_{2}$ muscarinic receptor knockout mice. This reduction in contractile activity was characterized by decreases in potency and maximal response, but not always both. Similar results were obtained with isoproterenol $(1.0 \mu \mathrm{M})$. The relaxant effects of forskolin in ileum, trachea and urinary bladder from $\mathrm{M}_{2}$ receptor knockout mice were approximately three- to nine-fold greater than those observed in the same tissues from wild type mice. Similar results were obtained with isoproterenol in ileum and urinary bladder, although the differences between wild type and $\mathrm{M}_{2}$ receptor knockout tissues were less than those observed with forskolin. In contrast, there was no significant difference between the relaxant effect of isoproterenol in trachea from wild type and $\mathrm{M}_{2}$ receptor knockout mice. In contrast to the results observed with oxotremorine-M as the contractile agent, forskolin and isoproterenol did not exhibit greater relaxant activity against KCl-induced contractions in $\mathrm{M}_{2}$ receptor knockout mice compared with wild type mice. These results suggest that a component of the contractile response to muscarinic agonists in smooth muscle involves an $\mathrm{M}_{2}$ muscarinic receptor-mediated inhibition of the relaxant effects of agents that increase cAMP levels. 
Muscarinic $\mathrm{M}_{2}$ and $\mathrm{M}_{3}$ receptors are abundantly expressed postjunctionally in smooth muscle where they mediate the contractile effects of acetylcholine (see reviews by Eglen et al. (1996) and Ehlert et al. (1997)). When measured in the absence of other heterologous agents, the contractile response to muscarinic agonists is inhibited by subtype selective antagonists in a manner consistent with an $M_{3}$ mechanism. This behavior is consistent with the known coupling of $M_{3}$ receptors to pertussis toxin-insensitive $G_{q}$, which mediates phosphoinositide hydrolysis and mobilization of $\mathrm{Ca}^{2+}$ (Candell et al., 1990; Noronha-Blob et al., 1989; Peralta et al., 1988; Roffel et al., 1990). The muscarinic $M_{2}$ receptor is known to signal through $G_{i}$ in smooth muscle to mediate pertussis toxin-sensitive responses including the inhibition of both adenylyl cyclase (Candell et al., 1990; Noronha-Blob et al., 1989; Yang et al., 1991) and $\mathrm{Ca}^{2+}$-activated potassium channels (Kotlikoff et al., 1992) as well as the stimulation of a nonselective cation conductance (Bolton and Zholos, 1997; Inoue and Isenberg, 1990). Ultimately, $\mathrm{M}_{2}$ mediated effects on contraction through these latter mechanisms are conditional upon $\mathrm{Ca}^{2+}$ mobilization via another receptor. Consequently, the pharmacological paradigms required to demonstrate a contractile role of the $\mathrm{M}_{2}$ receptor are more complicated than the standard assay used to establish a role for the $M_{3}$ receptor. Nevertheless, pharmacological studies employing pertussis toxin, irreversible $M_{3}$ selective muscarinic antagonists, and heterologous contractile and relaxant agents have demonstrated two roles for the $\mathrm{M}_{2}$ receptor in contraction - an inhibition of the relaxation caused by agents that increase cAMP and a conditional potentiation of the $\mathrm{M}_{3}$ receptormediated contractions (Hegde et al., 1997; Sawyer and Ehlert, 1998; Sawyer and Ehlert, 1999b; Shen and Mitchelson, 1998; Thomas et al., 1993; Thomas and Ehlert, 1994; Thomas and Ehlert, 1996).

Recent studies on $\mathrm{M}_{2}$ and $\mathrm{M}_{3}$ receptor knockout mice are generally consistent with the results of pharmacological experiments on wild type animals of other species showing that it is primarily the $M_{3}$ receptor that mediates a direct contraction in smooth muscle. In $\mathrm{M}_{3}$ receptor knockout mice, the muscarinic contractile response of the ileum and urinary bladder are greatly reduced compared to wild type mice (Matsui et al., 2000; Stengel et al., 2002), whereas a much smaller decrement in contractile function was noted in $\mathrm{M}_{2}$ receptor knockout mice (Stengel et al., 2000). In mutant mice lacking both $M_{2}$ and $M_{3}$ receptors, the potent contractile response to muscarinic agonists in ileum and urinary bladder is completely eliminated, demonstrating that only $\mathrm{M}_{2}$ and $\mathrm{M}_{3}$ muscarinic receptors contribute to the direct contractile response in these tissues (Matsui et al., in press). So far, 
no studies have been reported on the role of $\mathrm{M}_{2}$ receptors in mediating an inhibition of the relaxant effects of agents that increase cAMP in muscarinic receptor knockout mice.

In this report, we describe the relaxant effects of isoproterenol and forskolin on muscarinic agonistinduced contraction of smooth muscle in wild type and $\mathrm{M}_{2}$ receptor knockout mice. Our results show that the relaxant effects of forskolin on muscarinic agonist-induced contractions, but not those elicited by $\mathrm{KCl}$, are greatly increased in the ileum, bladder and trachea of $\mathrm{M}_{2}$ receptor knockout mice as compared with wild type mice. Under similar conditions, the relaxant effect of isoproterenol was also enhanced in the ileum and urinary bladder, but not in the trachea. These results demonstrate that a component of the contractile mechanism of muscarinic agonists in smooth muscle involves an $\mathrm{M}_{2}$ receptor-mediated inhibition of the relaxant effects of agents that increase cAMP levels. 


\section{METHODS}

Animals: The generation of $\mathrm{M}_{2}$ muscarinic receptor knockout mice has been described previously (Matsui et al., in press). A mutant mouse line was established in a mixed background between 129/SvJ and C57BL/6. This line was backcrossed with C57BL/6 mice to yield an N3 generation of $\mathrm{M}_{2}-/-$ muscarinic receptor knockout mice, which were used in the pharmacological studies described in this report. C57BL/6 mice were purchased from Harlan, Indianapolis, Indiana.

Isolated smooth muscle: Wild type $\left(\mathrm{M}_{2}+/+\right)$ male $\mathrm{C} 57 \mathrm{BL} / 6$ mice and male $\mathrm{M}_{2}$ muscarinic receptor knockout $\left(\mathrm{M}_{2}-\right.$ /-) mice were used in these studies. Their body weights were approximately $25-30 \mathrm{~g}$. The mice were euthanized by $\mathrm{CO}_{2}$ asphyxiation and various smooth muscle preparations were immediately removed. Segments of whole ileum $(1.5-2 \mathrm{~cm}$ in length) were excised starting at a point approximately $5 \mathrm{~cm}$ rostral from the ileoceacal junction and mounted longitudinally in an organ bath with silk thread. The whole trachea was dissected free of adhering tissue and mounted as a single ring on stainless steel supports. The whole urinary bladder was removed and mounted longitudinally with silk thread. All tissues were bathed at $37^{\circ} \mathrm{C}$ within $50 \mathrm{ml}$ organ baths containing Krebs Ringer Bicarbonate (KRB) buffer $\left(\mathrm{NaCl}, 124 \mathrm{mM} ; \mathrm{KCl}, 5.0 \mathrm{mM} ; \mathrm{MgSO}_{4}, 1.8 \mathrm{mM} ; \mathrm{NaHCO}_{3}, 26 \mathrm{mM}\right.$; $\mathrm{KH}_{2} \mathrm{PO}_{4}, 1.2 ; \mathrm{CaCl}_{2}, 1.8 \mathrm{mM}$; glucose, $\left.10 \mathrm{mM}\right)$ gassed with $\mathrm{O}_{2} / \mathrm{CO}_{2}(19 / 1)$. Indomethacin $(1.0 \mu \mathrm{M})$ was present in the KRB buffer at all times. Indomethacin is often included in experiments on tracheal smooth muscle to remove the influence of endogenous prostaglandins (Muccitelli et al., 1987), to dissipate spontaneous tracheal tone (Small et al., 1990), and to maintain receptor-stimulated tone (Mansour and Daniel, 1986), although the reasons for these uses are incompletely understood. To maintain consistency, all tissues were exposed to indomethacin. The tissues were connected to force-displacement transducers and isometric tension was recorded using either a Polygraph (Grass Instruments, Quincy, Massachusetts) or PowerLab (ADInstruments, Grand Junction, Colorado) recording systems. Resting tensions were adjusted to loads equivalent to those generated by masses of $0.3,2$ and $0.5 \mathrm{~g}$ in the ileum, trachea and urinary bladder, respectively. The tissues were first allowed to equilibrate for at least $60 \mathrm{~min}$, and then three test doses of $\mathrm{KCl}(50 \mathrm{mM})$ were applied. After each test dose, the tissues were washed with fresh KRB buffer and allowed to rest for approximately 5 to $10 \mathrm{~min}$. The contractile response to the third test dose of 
$\mathrm{KCl}$ was used in calculations to normalize the response to the muscarinic agonist, oxotremorine-M, relative to that elicited by $\mathrm{KCl}$. The tissues were allowed to rest for $15 \mathrm{~min}$, and then a cumulative concentration-response curve to oxotremorine-M was measured, with the agonist concentrations being spaced three-fold. Only the tonic phase of contraction was used in the calculation of concentration-response curves. The tissues were washed with fresh KRB buffer and allowed to rest for $30 \mathrm{~min}$. A second control concentration-response curve to oxotremorine-M was measured, and this second curve was used as the control to which the curves measured in the presence of relaxant agents were compared. Tissues were washed extensively with fresh KRB buffer after measurement of each concentration-response curve and were allowed to rest for $30 \mathrm{~min}$ before the next concentration-response curve was measured.

Calculations: The $E C_{50}$ value (concentration of agonist eliciting half-maximal contraction) and the maximal response $\left(E_{\max }\right)$ to oxotremorine-M were estimated from the concentration-response data by nonlinear regression analysis using an increasing logistic equation as described previously (Candell et al., 1990). In most cases, the relaxant agents caused a decrease in both the maximal response and potency of oxotremorine-M for eliciting contraction. In such instances, the effect of the relaxant agent could be simulated quantitatively by assuming that the relaxant agent caused a decrease in the proportion of receptors or the intrinsic efficacy of the receptoroxotremorine-M complex. We refer to this phenomenon as the decrease in the "observed coupling efficiency" of oxotremorine-M caused by the relaxant agent. This measure of relaxant action was calculated as described previously (Ostrom and Ehlert, 1997) using a method akin to Furchgott analysis (Furchgott, 1966). This estimate is simply an empirical measure of the relaxant effect, and no conclusion about the mechanism of relaxation is deduced with this estimate. We simply use the estimate of observed coupling efficiency as a means of summating the inhibitory effects of the relaxant agent on the two disparate parameters, $E C_{50}$ and $E_{\max }$. For each experiment, the estimates of $E C_{50}$ were converted to negative logarithms $\left(p E C_{50}\right)$, and the effect of the relaxant agent on the $E C_{50}$ value was calculated as the difference between the control $p E C_{50}$ value and that measured in the presence of the relaxant agent (i.e., $\log E C_{50}$ shift). To assess whether the relaxant agent had a significant effect on the $E C_{50}$ value, a t-distribution was used to determine if the $\log E C_{50}$ shift values were significantly different from zero. Similarly, a t-distribution was used to determine if the logarithm of the observed coupling efficiencies were significantly 
different from a value of zero (i.e., no change in coupling efficiency). A paired t-test was used to determine if the relaxant agent had a significant effect on the $E_{\max }$. To determine if these estimates of relaxant activity in $\mathrm{M}_{2}$ receptor knockout mice were significantly different from those measured in wild type animals, an unpaired t-test was used.

Drugs and chemicals: The reagents used in this study were obtained from the following sources. OxotremorineM, Research Biochemicals International, Natick, MA; isoproterenol, indomethacin and tetrodotoxin, Sigma Chemical Company, St. Louis, MO; forskolin, Calbiochem, La Jolla, CA. 


\section{RESULTS}

Oxotremorine-M elicited contractions in ileum, trachea and urinary bladder of wild type mice with mean $p E C_{50}$ values $\pm \mathrm{SEM}$ of $6.70 \pm 0.037,6.94 \pm 0.036$ and $6.58 \pm 0.044$ and $E_{\max }$ values, expressed as mass equivalents, of $1.36 \pm 0.083,2.60 \pm 0.23$ and $4.3 \pm 0.47 \mathrm{~g}$, respectively. When expressed relative to the contraction elicited by $\mathrm{KCl}(50 \mathrm{mM})$, the $E_{\max }$ values of oxotremorine-M in these tissues were $188 \pm 20,218 \pm 14$ and $223 \pm 11 \%$, respectively. In $\mathrm{M}_{2}$ receptor knockout mice, the potency of oxotremorine- $\mathrm{M}$ was less. This decrease in potency corresponded to mean $E C_{50}$ values that were 2.1-, 1.21- and 1.48-fold greater in the ileum, trachea and urinary bladder, respectively. These differences reached statistical significance in ileum $(\mathrm{P}=0.00013)$, but not in trachea $(\mathrm{P}=0.124)$ or urinary bladder $(\mathrm{P}=0.114)$. There were no significant differences between wild type and $\mathrm{M}_{2}$ receptor knockout mice with regard to the $E_{\max }$ values of oxotremorine-M expressed relative to the contraction elicited to $\mathrm{KCl}$ in the three tissues $(\mathrm{P}=0.93,0.12$ and 0.11 in ileum, trachea and urinary bladder, respectively). A similar conclusion was reached when $E_{\max }$ was expressed in units of mass equivalents. These data are summarized in Figures 1 and 2 and Table 1. In a limited number of experiments, we found that tetrodotoxin (1 $\mu \mathrm{M})$ was without effect on the contractile response to oxotremorine-M in wild type and $\mathrm{M}_{2}$ receptor knockout mice.

Figure 1 shows the relaxant effect of forskolin $(5.0 \mu \mathrm{M})$ on the contractile response to oxotremorine-M in ileum, trachea and urinary bladder. In wild type mice, forskolin caused a significant reduction in the potency of oxotremorine-M in each tissue (i.e., increase in $E C_{50}$ or decrease in $p E C_{50}$ ), and a significant reduction in $E_{\max }$ in the trachea, but not in ileum or urinary bladder. These changes resulted in a significant reduction in the observed coupling efficiency of oxotremorine-M in the three tissues. Similar results were obtained with forskolin in smooth muscle from $\mathrm{M}_{2}$ receptor knockout mice although the effects were greater. Forskolin caused 3.6-, 3.1- and 9.3-fold greater reductions in the coupling efficiency of oxotremorine- $\mathrm{M}$ in the ileum, trachea and urinary bladder of $\mathbf{M}_{2}$ receptor knockout mice as compared with wild type mice. These results are summarized in Table 2.

Similar results were obtained with isoproterenol $(1.0 \mu \mathrm{M})$ except that there was a smaller difference between the relaxant effects of isoproterenol in wild type and $\mathrm{M}_{2}$ receptor knockout mice (see Figure 2). The net effect of isoproterenol was to cause a significant reduction in the coupling efficiency of oxotremorine-M in the three tissues in both wild type and $\mathrm{M}_{2}$ receptor knockout mice. Isoproterenol elicited 2.0- and 2.3-fold greater 
reductions in the observed coupling efficiency of oxotremorine-M in ileum and urinary bladder from $\mathrm{M}_{2}$ receptor knockout mice as compared to wild type mice (see Table 3). In contrast, the relaxant effects of isoproterenol in trachea were practically the same in wild type and $\mathrm{M}_{2}$ receptor knockout mice (i.e., no significant differences between wild type and $\mathrm{M}_{2}$ knockout with regard to the effect of isoproterenol on $E_{\text {max }}$, shift in $E C_{50}$ or observed coupling efficiency, $\mathrm{P}=0.54,0.39$ and 0.29 , respectively). The mean values for the effects of isoproterenol on $E_{\text {max }}, E C_{50}$ and observed coupling efficiency in the trachea showed greater relaxant effects in wild type mice compared with $\mathrm{M}_{2}$ receptor knockout mice, although these differences between groups were not statistically significant. These results are summarized in Table 3.

Figure 3 shows the relaxant effects of forskolin $(5.0 \mu \mathrm{M})$ and isoproterenol $(1.0 \mu \mathrm{M})$ on the contractile response elicited by $\mathrm{KCl}(50 \mathrm{mM})$. Forskolin caused a significant inhibition of $\mathrm{KCl}$-induced contractions in ileum (55\%), trachea (84\%) and urinary bladder (39\%) from wild type mice. However, in contrast to that observed with oxotremorine- $\mathrm{M}$ as the contractile agent, the relaxant effects of forskolin against $\mathrm{KCl}$-induced contractions were not significantly different between wild type and $\mathrm{M}_{2}$ receptor knockout mice in ileum $(P=0.78)$, trachea $(P=$ 0.073) and urinary bladder $(\mathrm{P}=0.84)$. There was a substantial, though statistically not significant, difference in the trachea; this change was in the direction of forskolin having a larger inhibitory effect in wild type trachea (84\%) compared with that from $\mathrm{M}_{2}$ receptor knockout trachea (76\%). Similar results were obtained when isoproterenol was used as the relaxant agent against $\mathrm{KCl}$-induced contractions. In wild type mice, isoproterenol caused a highly significant inhibition of $\mathrm{KCl}$-induced contractions in ileum (39\%), trachea (82\%) and urinary bladder (54\%). In ileum and urinary bladder, there were no significant differences in the effects of isoproterenol on $\mathrm{KCl}$-induced contractions between wild type and $\mathrm{M}_{2}$ receptor knockout mice $(\mathrm{P}=0.95$ and 0.99 in ileum and urinary bladder, respectively). In trachea, isoproterenol had similar relaxant effects against $\mathrm{KCl}$-induced contractions in the two types of mice, however, the relaxant effect of isoproterenol was slightly yet significantly greater in wild type mice as compared with $\mathrm{M}_{2}$ receptor knockout mice $(\mathrm{P}=0.039)$. Thus, in contrast to the results of the experiments in which oxotremorine-M was used as the contractile agent, the relaxant effects of isoproterenol and forskolin against $\mathrm{KCl}$-induced contractions in $\mathrm{M}_{2}$ receptor knockout mice were equal to or less than those observed in wild type mice. 
We also investigated the effects of the potassium channel activator, pinacidil, on the contractile response to oxotremorine-M in smooth muscle from wild type and $\mathrm{M}_{2}$ receptor knockout mice (see Table 4). Pinacidil caused a decrease in the potency of oxotremorine-M in ileum, trachea and urinary bladder and a small increase in $E_{\max }$ in both wild type and $\mathrm{M}_{2}$ receptor knockout mice. However, there were no significant differences between the effects of pinacidil in wild type and $\mathrm{M}_{2}$ receptor knockout mice. 


\section{DISCUSSION}

Our observations on the contractile action of the selective muscarinic agonist, oxotremorine-M, in trachea and urinary bladder of $\mathrm{M}_{2}$ muscarinic receptor knockout mice are similar to those reported by Stengel et al. (2000) using the nonselective cholinergic agonist carbachol. We found that the potency of oxotremorine-M decreased 1.21- and 1.48-fold in trachea and urinary bladder from $\mathrm{M}_{2}$ receptor knockout mice, respectively, compared with wild type, whereas Stengel and cowerkers (2000) observed 1.9- and 1.6-fold reductions in potency in these tissues with carbachol. We also observed a significant 2.1-fold reduction in the potency of oxotremorine-M in ileum in $\mathrm{M}_{2}$ receptor knockout mice compared with wild type. These results suggest that, in wild type mice, the $\mathrm{M}_{2}$ receptor plays some modulatory roles although the $\mathrm{M}_{3}$ receptor is capable of eliciting most of the direct contractile response to muscarinic agonists in these tissues. Moreover, Matsui and coworkers (in press) showed that the direct muscarinic contractile response is completely eliminated in ileum and urinary bladder from mutant mice lacking both $\mathrm{M}_{2}$ and $\mathrm{M}_{3}$ receptors. These results show that the postjunctional effects of muscarinic agonists can be attributed almost entirely to $\mathrm{M}_{2}$ and $\mathrm{M}_{3}$ muscarinic receptors.

Prior studies on gastrointestinal (Candell et al., 1990; Sawyer and Ehlert, 1998; Zhang and Buxton, 1991), tracheal (Yang et al., 1991) and urinary bladder smooth muscle (Noronha-Blob et al., 1989) have shown that the $\mathrm{M}_{2}$ muscarinic receptor mediates an inhibition of adenylyl cyclase. In ileum and trachea, this effect has been shown to be prevented by pertussis toxin-treatment (Sankary et al., 1988; Thomas and Ehlert, 1994). In contrast, pertussis toxin-treatment has no inhibitory effect on $\mathrm{M}_{3}$ receptor-mediated phosphoinositide hydrolysis in gastrointestinal or tracheal smooth muscle, or on the contractile response to muscarinic agonists in guinea pig ileum, colon or trachea (Ostrom and Ehlert, 1999; Sawyer and Ehlert, 1999a; Sawyer and Ehlert, 1999b; Thomas and Ehlert, 1994; Thomas and Ehlert, 1996). This result suggests that the $M_{2}$ receptor has little role in mediating the potent contractile response to muscarinic agonists in guinea pig smooth muscle, because uncoupling $\mathrm{M}_{2}$ receptor signaling with pertussis toxin does not inhibit contraction. However, pertussis toxin-treatment has been shown to enhance the relaxant effects of forskolin on oxotremorine-M-mediated contractions in ileum and trachea (Ostrom and Ehlert, 1997; Ostrom and Ehlert, 1998). Presumably, in the presence of forskolin, part of the contractile mechanism of oxotremorine-M involves an $\mathrm{M}_{2}$ receptor-mediated inhibition of the relaxant effect of 
forskolin. This mechanism probably involves an $\mathrm{M}_{2}$ receptor-mediated inhibition of adenylyl cyclase because forskolin is thought to elicit relaxation in smooth muscle through cAMP (see review, Berridge (1975)). By uncoupling this $\mathrm{M}_{2}$ mechanism with pertussis toxin, the muscarinic contractile response is now more susceptible to inhibition by forskolin. This interpretation is consistent with the observation that pertussis toxin is without effect on forskolin-mediated inhibition of histamine-induced contractions (Ostrom and Ehlert, 1997; Ostrom and Ehlert, 1998). Since histamine elicits contraction in smooth muscle through activation of $H_{1}$ receptors (Black et al., 1972), which signal through $G_{q}$ without activation of $G_{i}$ (Arrang et al., 1995), one would not expect pertussis toxin to influence histamine-induced contractions or their inhibition by forskolin. Our results in $\mathrm{M}_{2} \mathrm{KO}$ mice with forskolin are consistent with our previous work in guinea pigs using pertussis toxin to inactivate $\mathrm{M}_{2}$ receptor signaling.

We have also observed that pertussis toxin-treatment enhances the relaxant effect of isoproterenol against oxotremorine-M-induced contraction of the guinea pig ileum, but not those elicited in the trachea (Ostrom and Ehlert, 1997; Ostrom and Ehlert, 1998; Thomas and Ehlert, 1994). As observed with forskolin, pertussis toxintreatment has no effect on the ability of isoproterenol to inhibit histamine-induced contraction in guinea pig ileum and trachea. Our results on $\mathrm{M}_{2}$ receptor knockout mice are consistent with these observations. We observed a significant increase in the relaxant action of isoproterenol against oxotremorine-M-induced contraction in ileum and urinary bladder from $\mathrm{M}_{2}$ receptor knockout mice, but not in trachea. Collectively, our results suggest that pertussis toxin is a useful tool for exploring the role of $\mathrm{M}_{2}$ receptors in smooth muscle.

It seems unlikely that the increased relaxant effectiveness of forskolin and isoproterenol in $\mathrm{M}_{2}$ muscarinic receptor knockout mice is due to an increase in the sensitivity of smooth muscle to relaxant agents. Our experiments utilizing $\mathrm{KCl}$ as the contractile stimulus showed that there was no increase in the sensitivity of ileum, trachea or urinary bladder to the relaxant effects of forskolin and isoproterenol in $\mathrm{M}_{2}$ receptor knockout mice compared with wild type. Moreover, we found that there was no increase in the relaxant action of the potassium channel activator, pinacidil, against oxotremorine-M-induced contraction in $\mathrm{M}_{2}$ receptor knockout mice. Since the relaxant mechanism of pinacidil does not involve cAMP, one would not expect to observe a change in the ability of pinacidil to inhibit oxotremorine-M-induced contractions in $\mathrm{M}_{2}$ receptor knockout mice. Thus, our studies with $\mathrm{KCl}$ and pinacidil in $\mathrm{M}_{2}$ receptor knockout mice are consistent with the postulate that the increased relaxant action 
of forskolin and isoproterenol against oxotremorine-M-induced contractions is due to the loss of $\mathbf{M}_{2}$ receptors and not to an increase in the relaxant effectiveness of forskolin and isoproterenol.

The pattern of changes in $\mathbf{M}_{2}$ muscarinic receptor knockout mice observed in this study show close agreement with prior pharmacological studies in wild type guinea pigs. One powerful method for investigating the function of $\mathrm{M}_{2}$ receptors in wild type smooth muscle involves inactivating all non- $\mathrm{M}_{2}$ muscarinic receptors with the aziridinium ion of 4-DAMP mustard (N-(2-chloroethyl)-4-piperidinyl diphenylacetic acid). Following this treatment, the muscarinic contractile response is measured in the presence of histamine and forskolin. Under these conditions, the contractile response to oxotremorine exhibits an $\mathrm{M}_{2}$ profile for pharmacological antagonism in the ileum and trachea (Thomas et al., 1993; Thomas and Ehlert, 1996). Presumably, the contractile mechanism of oxotremorine-M under these conditions involves an $\mathrm{M}_{2}$ receptor-mediated inhibition of the relaxant effect of forskolin on histamine-induced contractions. When, isoproterenol is used in the paradigm in place of forskolin in the ileum, the potency of oxotremorine-M is less, and the pharmacological antagonism exhibits a profile midway between $\mathrm{M}_{2}-$ and $\mathrm{M}_{3}$-like (Thomas et al., 1993). Analogous types of experiments with isoproterenol in guinea pig urinary bladder (Hegde et al., 1997) and with isoproterenol and forskolin in guinea pig taenia caeci (Shen and Mitchelson, 1998) have yielded similar results. These data suggest that the $M_{2}$ receptor has a smaller role in inhibiting the relaxant effects of isoproterenol as compared to those of forskolin. Moreover, in the trachea, this type of experimental paradigm shows no role for the $\mathrm{M}_{2}$ receptor in opposing the relaxant effects of isoproterenol on histamine-induced contractions (Ostrom and Ehlert, 1998; Ostrom and Ehlert, 1999). These prior pharmacological studies are consistent with the present data on $\mathrm{M}_{2}$ receptor knockout mice. As described above, we found a greater increase in the relaxant effectiveness of forskolin compared with isoproterenol against oxotremorine-M-induced contractions in ileum from $\mathrm{M}_{2}$ receptor knockout mice as compared with wild type. Also, we found no difference between trachea from wild type and $\mathrm{M}_{2}$ receptor knockout mice with regard to the relaxant effectiveness of isoproterenol against oxotremorine-M-induced contractions. These results indicate that the $\mathrm{M}_{2}$ receptor does not oppose isoproterenol-induced relaxation in the trachea.

It has been suggested that the lack of the role of the $\mathrm{M}_{2}$ receptor in opposing isoproterenol-induced relaxation in the trachea implies that the relaxant mechanism of isoproterenol in the trachea is through a noncAMP mechanism (Ostrom and Ehlert, 1998; Ostrom and Ehlert, 1999). Torphy (1994) has previously proposed a 
non-cAMP mechanism, which could involve stimulation of $\mathrm{Ca}^{2+}$ activated $\mathrm{K}^{+}$channels. In bovine trachea, it has been shown that muscarinic receptor activation inhibits the increase in cAMP levels elicited by both forskolin and isoproterenol (Ostrom and Ehlert, 1998). Also, when cAMP levels and relaxation of muscarinic agonist-induced contractions are measured under identical conditions, forskolin-induced relaxation obeys a saturable, increasing function of the cytosolic concentration of cAMP, suggesting a functional relationship between the two (Ostrom and Ehlert, 1998). In contrast, no functional relationship between relaxation and the cytosolic concentration of cAMP was observed for isoproterenol (Ostrom and Ehlert, 1998). An increase in relaxation from 30 to $100 \%$ occurred with no change in the cAMP concentration. The smaller role of the $\mathrm{M}_{2}$ receptor in opposing isoproterenol-induced relaxation in the ileum compared with that caused by forskolin suggests that some, but not all, of the relaxation caused by isoproterenol in the ileum is mediated through cAMP. This could be explained if the $\mathrm{G}_{\mathrm{s}}$ activated by $\beta$-adrenergic receptors acts through at least two pathways - adenylyl cyclase as well as another pathway that is unopposed by $\mathrm{M}_{2}$ receptor activation. Forskolin, which acts downstream at the level of adenylyl cyclase, would be unable to elicit such a non-cAMP dependent mechanism for relaxation.

Prior studies in guinea pigs have shown that, in addition to opposing cAMP-mediated relaxation, $\mathrm{M}_{2}$ receptor activation also causes a conditional potentiation of $\mathrm{M}_{3}$ muscarinic receptor-mediated contractions (Sawyer and Ehlert, 1999b). This conclusion was based on the unique pattern of sensitivity of the muscarinic contractile response in the colon to pertussis toxin and both competitive and irreversible muscarinic antagonists. The data were consistent with the postulate that $\mathrm{M}_{2}$ receptors have no direct contractile action by themselves, but cause a conditional potentiation of $\mathrm{M}_{3}$ receptor-mediated contractions. As suggested previously (Ehlert, in press; Ehlert et al., 1999; Ehlert et al., 1997; Sawyer and Ehlert, 1999b), a possible mechanism for this action could be $\mathrm{M}_{2}$ receptor-meditated stimulation of the non-selective cation conductance or inhibition of $\mathrm{Ca}^{2+}$-activated $\mathrm{K}^{+}$channels. Both of these mechanisms are $\mathrm{Ca}^{2+}$-dependent; hence, they fulfill the necessary criterion of being conditional upon $\mathrm{Ca}^{2+}$ mobilization by the $\mathrm{M}_{3}$ receptor. At first, it might seem that a loss of these $\mathrm{M}_{2}$ responses could account for the increased susceptibility of the muscarinic contractile response to relaxant agents in $\mathrm{M}_{2}$ receptor knockout mice. However, our method of analysis makes this possibility unlikely. Our null method approach compares equivalent contractile stimuli in the presence and absence of the relaxant agents. There is no inherent reason to assume that a direct contractile stimulus mediated through an interaction between $\mathrm{M}_{2}$ and $\mathrm{M}_{3}$ receptors in wild type mice would 
be more resistant to relaxant agents than an equivalent stimulus activated only by $M_{3}$ receptors in $M_{2}$ receptor knockout mice. Moreover, if loss of the latter mechanism were responsible for the increased relaxant action of forskolin and isoproterenol in $\mathrm{M}_{2}$ receptor knockout mice, then, for a given tissue, we would have expected to observe the same increase in the relaxant effectiveness of both forskolin and isoproterenol. However, this was not observed as described above. In contrast, if the $\mathbf{M}_{2}$ receptor mediates an attenuation of the relaxant stimulus directly (e.g., through inhibition of adenylyl cyclase), this mechanism could explain why the relaxant activity of forskolin and isoproterenol increased differentially in tissues from $\mathbf{M}_{2}$ receptor knockout mice. Finally, it is important to mention that our null method also controls for compensatory changes in the activity of $\mathbf{M}_{3}$ receptors in $\mathrm{M}_{2}$ receptor knockout mice. Future studies on $\mathrm{M}_{2}$ receptor knockout mice may help to sort out the mechanism of the $\mathrm{M}_{2}$ receptor-mediated potentiation of $\mathrm{M}_{3}$ receptor mediated contractions. 


\section{ACKNOWLEDGEMENTS}

We thank T. Tamai, T. Ishikawa and K. Takaku for technical advice; I. Ishii, A. Matsunaga and A. Yokoi for blastocyst injections; Y. Araki, S. Kobayashi, N. Matsubara, H. Karasawa, D. Motomura and S. Takahashi for technical assistance; and T. Manabe for his continuous encouragement. 


\section{REFERENCES}

Arrang JM, Drutel G, Garbarg M, Ruat M, Traiffort E and Schwartz JC (1995) Molecular and functional diversity of histamine receptor subtypes. Ann. New York Acad. Sci. 757: 314-23.

Berridge MJ (1975) The interaction of cyclic nucleotides and calcium in the control of cellular activity. Adv. Cyclic Nucleotide Res. 6: 1-98.

Black JW, Duncan WA, Durant CJ, Ganellin CR and Parsons EM (1972) Definition and antagonism of histamine H 2 -receptors. Nature 236: 385-90.

Bolton TB and Zholos AV (1997) Activation of M2 muscarinic receptors in guinea-pig ileum opens cationic channels modulated by M3 muscarinic receptors. Life Sci. 60: 1121-8.

Candell LM, Yun SH, Tran LL and Ehlert FJ (1990) Differential coupling of subtypes of the muscarinic receptor to adenylate cyclase and phosphoinositide hydrolysis in the longitudinal muscle of the rat ileum. Mol. Pharmacol. 38: 689-97.

Eglen RM, Hegde SS and Watson N (1996) Muscarinic receptor subtypes and smooth muscle function. Pharmacol. Rev. 48: 531-565.

Ehlert FJ. (in press). Pharmacological analysis of the contractile role of $\mathrm{M}_{2}$ and $\mathrm{M}_{3}$ muscarinic receptor in smooth muscle. In T. Kenakin (Ed.), Receptors and Channels, : Harwood Academic Publishers.

Ehlert FJ, Sawyer GW and Esqueda EE (1999) Contractile role of M2 and M3 muscarinic receptors in gastrointestinal smooth muscle [published erratum appears in Life Sci 1999;64(26):2535]. Life Sci. 64: $387-94$.

Ehlert FJ, Thomas EA, Gerstin EH and Griffin MT. (1997). Muscarinic receptors and gastrointestinal smooth muscle. In R. M. Eglen (Ed.), Muscarinic Receptor Subtypes in Smooth Muscle, (pp. 92-147). Boca Raton: CRC Press.

Furchgott RF (1966) The use of $\beta$-haloalkylamines in the differentiation of receptors and in the determination of dissociation constants of receptor-agonist complexes. Adv. Drug Res. 3: 21-55. 
Hegde SS, Choppin A, Bonhaus D, Briaud S, Loeb M, Moy TM, Loury D and Eglen RM (1997) Functional role of M2 and M3 muscarinic receptors in the urinary bladder of rats in vitro and in vivo. Brit. J. Pharmacol. 120: $1409-18$.

Inoue R and Isenberg G (1990) Acetylcholine activates nonselective cation channels in guinea pig ileum through a G protein. Amer. J. Physiol. 258: C1173-8.

Kotlikoff MI, Kume H and Tomasic M (1992) Muscarinic regulation of membrane ion channels in airway smooth muscle cells. Biochem. Pharmacol. 43: 5-10.

Mansour S and Daniel EE (1986) Maintenance of tone, role of arachidonate metabolites, and effects of sensitization in guinea pig trachea. Can J Physiol Pharmacol 64: 1096-103.

Matsui M, Motomura D, Fujikawa T, Jiang J, Takahashi S and Taketo $\mathbf{M}$ (in press) Mice lacking $\mathbf{M}_{2}$ and $\mathbf{M}_{3}$ muscarinic acetylcholine receptors are devoid of cholinergic smooth muscle contractions but still viable. J. Neurosci.

Matsui M, Motomura D, Karasawa H, Fujikawa T, Jiang J, Komiya Y, Takahashi S and Taketo MM (2000) Multiple functional defects in peripheral autonomic organs in mice lacking muscarinic acetylcholine receptor gene for the M3 subtype. Proc. Natl. Acad. Sci. U.S.A. 97: 9579-84.

Muccitelli RM, Tucker SS, Hay DW, Torphy TJ and Wasserman MA (1987) Is the guinea pig trachea a good in vitro model of human large and central airways? Comparison on leukotriene-, methacholine-, histamineand antigen-induced contractions. J. Pharmacol. Exp. Ther. 243: 467-73.

Noronha-Blob L, Lowe V, Patton A, Canning B, Costello D and Kinnier WJ (1989) Muscarinic receptors: relationships among phosphoinositide breakdown, adenylate cyclase inhibition, in vitro detrusor muscle contractions and in vivo cystometrogram studies in guinea pig bladder. J. Pharmacol. Exp. Ther. 249: 843-851.

Ostrom RS and Ehlert FJ (1997) $\mathrm{M}_{2}$ muscarinic receptor inhibition of agonist-induced cyclic adenosine monophosphate accumulation and relaxation in the guinea pig ileum. J. Pharmacol. Exp. Ther. 280: 1-11.

Ostrom RS and Ehlert FJ (1998) M2 muscarinic receptors inhibit forskolin- but not isoproterenol-mediated relaxation in bovine tracheal smooth muscle. J. Pharmacol. Exp. Ther. 286: 234-242. 
Ostrom RS and Ehlert FJ (1999) Comparison of functional antagonism between isoproterenol and M2 muscarinic receptors in guinea pig ileum and trachea. J. Pharmacol. Exp. Ther. 288: 969-976.

Peralta EG, Ashkenazi A, Winslow JW, Ramachandran J and Capon DJ (1988) Differential regulation of PI hydrolysis and adenylyl cyclase by muscarinic receptor subtypes. Nature 334: 434-7.

Roffel AF, Meurs H, Elzinga CR and Zaagsma J (1990) Characterization of the muscarinic receptor subtype involved in phosphoinositide metabolism in bovine tracheal smooth muscle. Brit. J. Pharmacol. 99: 2936.

Sankary RM, Jones CA, Madison JM and Brown JK (1988) Muscarinic cholinergic inhibition of cyclic AMP accumulation in airway smooth muscle. Role of a pertussis toxin-sensitive protein. Amer. Rev. Resp. Dis. 138: $145-50$.

Sawyer G and Ehlert F (1999a) Pertussis toxin increases isoproterenol induced relaxation in field-stimulated ileum [published erratum appears in Eur J Pharmacol 1999 May 21;372(3):329]. Eur. J. Pharmacol. 367: 81-4.

Sawyer GW and Ehlert FJ (1998) Contractile role of the M2 and M3 muscarinic receptors in the guinea pig colon. J. Pharmacol. Exp. Ther. 284: 269-277.

Sawyer GW and Ehlert FJ (1999b) Muscarinic M3 receptor inactivation reveals a pertussis toxin-sensitive contractile response in the guinea pig colon. J. Pharmacol. Exp. Ther. 289: 464-76.

Shen A and Mitchelson F (1998) Muscarinic M2 receptor-mediated contraction in the guinea pig Taenia caeci: possible involvement of protein kinase C. Biochem. Pharmacol. 56: 1529-37.

Small RC, Good DM, Dixon JS and Kennedy I (1990) The effects of epithelium removal on the actions of cholinomimetic drugs in opened segments and perfused tubular preparations of guinea- pig trachea. $\mathrm{Br} J$ Pharmacol 100: 516-22.

Stengel PW, Gomeza J, Wess J and Cohen ML (2000) M(2) and M(4) receptor knockout mice: muscarinic receptor function in cardiac and smooth muscle in vitro. J. Pharmacol. Exp. Ther. 292: 877-85.

Stengel PW, Yamada M, Wess J and Cohen ML (2002) M(3)-receptor knockout mice: muscarinic receptor function in atria, stomach fundus, urinary bladder, and trachea. Am J Physiol Regul Integr Comp Physiol 282: R1443-9. 
Thomas EA, Baker SA and Ehlert FJ (1993) Functional role for the M2 muscarinic receptor in smooth muscle of guinea pig ileum. Mol. Pharmacol. 44: 102-10.

Thomas EA and Ehlert FJ (1994) Pertussis toxin blocks M2 muscarinic receptor-mediated effects on contraction and cyclic AMP in the guinea pig ileum, but not M3-mediated contractions and phosphoinositide hydrolysis. J. Pharmacol. Exp. Ther. 271: 1042-50.

Thomas EA and Ehlert FJ (1996) Involvement of the M2 muscarinic receptor in contractions of the guinea pig trachea, guinea pig esophagus and rat fundus. Biochem. Pharmacol. 51: 779-788.

Torphy TJ (1994) Beta-adrenoceptors, cAMP and airway smooth muscle relaxation: challenges to the dogma. Trends Pharmacol. Sci. 15: 370-4.

Yang CM, Chou SP and Sung TC (1991) Muscarinic receptor subtypes coupled to generation of different second messengers in isolated tracheal smooth muscle cells. Brit. J. Pharmacol. 104: 613-8.

Zhang LB and Buxton IL (1991) Muscarinic receptors in canine colonic circular smooth muscle. II. Signal transduction pathways. Mol. Pharmacol. 40: 952-9. 


\section{FOOTNOTES}

a This work was supported by NIH Grant NS30882, by Grants-in-Aid for Scientific Research from the Ministry of Education, Science, Sports and Culture (M.M., M.M.T.), by Industrial Technology Research Grant Program in '00 and '02 from the New Energy and Industrial Technology Development Organization of Japan (M.M.), and by Grant from Organization for Pharmaceutical Safety and Research, Japan (M.M.T.).

Send reprint requests to:

Frederick J. Ehlert, Ph.D.

Department of Pharmacology

College of Medicine

University of California, Irvine

Irvine, California 92697-4625 
Table 1: $\quad$ Contractile activity of oxotremorine-M in smooth muscle from wild type and $\mathrm{M}_{2}$ muscarinic receptor knockout (KO) mice. ${ }^{a}$

Ileum Trachea $\quad$ Urinary Bladder

Wild Type $(n=5)$
$E_{\max }(\% \mathrm{KCl})$
$188 \pm 20$
$218 \pm 14$
$223 \pm 11$
$p E C_{50}$
$6.70 \pm 0.037$
$6.94 \pm 0.036$
$6.58 \pm 0.044$

$\underline{M}_{2}$ Receptor KO $(n=5)$
$E_{\max }(\% \mathrm{KCl})$
$185 \pm 22$
$191 \pm 6.1$
$188 \pm 17$
$p E C_{50}$
$6.38 \pm 0.028^{b}$
$6.86 \pm 0.037$
$6.41 \pm 0.055$

a The data were calculated from the experiments shown in Figures 1 and 2. Mean values \pm SEM from five wild type and five $\mathrm{M}_{2}$ receptor knockout mice are shown.

b Significantly different from that measured in wild type mice, $\mathrm{P}=0.00013$. 
Table 2: Effect of forskolin on the contractile response to oxotremorine-M in smooth muscle from wild type and $\mathrm{M}_{2}$ muscarinic receptor knockout (KO) mice. ${ }^{\mathrm{a}}$

Ileum

Trachea

Urinary Bladder

Wild Type $(n=5)$

Forskolin
$E_{\max }(\%$ control $)$
$90 \pm 3.9$
$63 \pm 5.2^{\mathrm{d}}$
$103 \pm 2.7$
$E C_{50}$ Shift (fold $)^{b}$
$1.6^{\mathrm{d}}$
$6.7^{\mathrm{d}}$
$2.2^{\mathrm{d}}$
$(0.20 \pm 0.032)$
$(0.83 \pm 0.057)$
$(0.35 \pm 0.053)$
$C E_{\text {observed }}{ }^{c}$
$0.57^{\mathrm{d}}$
$0.098^{\mathrm{d}}$
$0.46^{\mathrm{d}}$
$(-0.25 \pm 0.033)$
$(-1.01 \pm 0.026)$
$(-0.34 \pm 0.058)$

\author{
$\underline{M}_{2}$ Receptor $K O(n=5)$ \\ Forskolin \\ $E_{\max }(\%$ control $)$ \\ $E C_{50}$ Shift (fold) ${ }^{b}$ \\ $C E_{\text {observed }}{ }^{c}$
}

$70 \pm 13$

$63 \pm 3.1^{d}$

$74 \pm 13$

$4.9^{\text {d.e }}$

$(0.69 \pm 0.055)$

$20^{\mathrm{d}, \mathrm{e}}$

$16^{\mathrm{d}, \mathrm{e}}$

$(1.30 \pm 0.018)$

$(1.20 \pm 0.16)$

$0.16^{\mathrm{d}, \mathrm{e}}$

$0.032^{\mathrm{d}, \mathrm{e}}$

$0.049^{\mathrm{d}, \mathrm{e}}$

$(-0.80 \pm 0.14)$

$(-1.50 \pm 0.029)$

$(-1.31 \pm 0.11)$

a The data were calculated from the experiments shown in Figure 1. Mean values \pm SEM from five wild type and five $\mathrm{M}_{2}$ receptor knockout mice are shown.

b Denotes the $E C_{50}$ value of oxotremorine-M measured in the presence of the relaxant agent divided by that measured in its absence. The values in parentheses beneath each estimate represent the mean $\log E C_{50}$ shift values \pm SEM.

c Denotes the observed coupling efficiency of oxotremorine-M in the presence of the relaxant agent relative to that in the absence of the relaxant agent. The values in parentheses beneath each estimate represent the mean $\log C E_{\text {observed }}$ values $\pm \mathrm{SEM}$.

d A significant effect of the relaxant agent relative to control. The probability values for these differences are as follows. Wild type mice, effects of forskolin: $E_{\max }$ in trachea, $\mathrm{P}=0.0020 ; E C_{50}$ shift in ileum, trachea and urinary bladder, $\mathrm{P}=0.0079,0.00013$ and $0.0028 ; C E_{\text {observed }}$ in ileum, trachea and urinary bladder, $\mathrm{P}=0.0023$, 
$7.0 \times 10^{-9}$ and $0.0012 . \mathrm{M}_{2}$ muscarinic receptor knockout mice, effects of forskolin: $E_{\max }$ in trachea, $\mathrm{P}=$ 0.00030; $E C_{50}$ shift in ileum, trachea and urinary bladder, $\mathrm{P}=0.0011,2.1 \times 10^{-7}$ and $0.0016 ; C E_{\text {observed }}$ in ileum, trachea and urinary bladder, $\mathrm{P}=0.00046,1.8 \times 10^{-10}$ and $1.3 \times 10^{-7}$.

Significantly different from the relaxant effect observed in wild type mice. The probability values for theses differences are as follows: Effects of forskolin on $E C_{50}$ shift in ileum, trachea and urinary bladder, $\mathrm{P}=$ 0.00027, 0.000047, and 0.0021; effects of forskolin on $C E_{\text {observed }}$ in ileum, trachea and urinary bladder, $\mathrm{P}=$ $0.00090,2.9 \times 10^{-6}$ and 0.00086 . 
Table 3: Effect of isoproterenol on the contractile response to oxotremorine-M in smooth muscle from wild type and $\mathrm{M}_{2}$ muscarinic receptor knockout $(\mathrm{KO})$ mice. ${ }^{\mathrm{a}}$

Ileum

Trachea

Urinary Bladder

Wild Type $(n=5)$

Isoproterenol
$E_{\max }(\%$ control $)$
$83 \pm 5.0^{\mathrm{d}}$
$74 \pm 4.4^{\mathrm{d}}$
$107 \pm 4.9$
$E C_{50}$ Shift (fold $)^{b}$
$3.1^{\mathrm{d}}$
$14.1^{\mathrm{d}}$
$1.8^{\mathrm{d}}$
$(0.49 \pm 0.061)$
$(1.15 \pm 0.098)$
$(0.26 \pm 0.059)$
$C E_{\text {observed }}{ }^{c}$
$0.28^{\mathrm{d}}$
$0.055^{\mathrm{d}}$
$0.62^{\mathrm{d}}$
$(-0.55 \pm 0.060)$
$(-1.26 \pm 0.082)$
$(-0.21 \pm 0.065)$

\section{$\underline{M}_{2}$ Receptor KO $(n=5)$ \\ Isoproterenol \\ $E_{\max }(\%$ control) \\ $E C_{50}$ Shift $(\text { fold })^{b}$ \\ $C E_{\text {observed }}{ }^{c}$}

$63 \pm 2.8^{\mathrm{d}, \mathrm{e}}$

$78 \pm 5.9^{d}$

$106 \pm 3.5$

$4.5^{\mathrm{d}}$

$(0.66 \pm 0.065)$

$11.4^{\mathrm{d}}$

$4.0^{\mathrm{d}, \mathrm{e}}$

$0.14^{\mathrm{d}, \mathrm{e}}$

$(1.06 \pm 0.023)$

$(0.61 \pm 0.063)$

$(-0.85 \pm 0.066)$

$0.070^{\mathrm{d}}$

$0.27^{\mathrm{d}, \mathrm{e}}$

$(-1.15 \pm 0.052)$

$(-0.56 \pm 0.057)$

a The data were calculated from the experiments shown in Figure 2. Mean values \pm SEM from five wild type and five $\mathrm{M}_{2}$ receptor knockout mice are shown.

b Denotes the $E C_{50}$ value of oxotremorine-M measured in the presence of the relaxant agent divided by that measured in its absence. The values in parentheses beneath each estimate represent the mean $\log E C_{50}$ shift values \pm SEM.

c Denotes the observed coupling efficiency of oxotremorine-M in the presence of the relaxant agent relative to that in the absence of the relaxant agent. The values in parentheses beneath each estimate represent the mean $\log C E_{\text {observed }}$ values $\pm \mathrm{SEM}$.

d A significant effect of the relaxant agent relative to control. The probability values for these differences are as follows. Wild type mice, effects of isoproterenol: $E_{\max }$ in ileum and trachea, $\mathrm{P}=0.030$ and $0.0038 ; E C_{50}$ shift in ileum, trachea and urinary bladder, $\mathrm{P}=0.0014,0.000030$ and $0.0068 ; C E_{\text {observed }}$ in ileum, trachea and urinary 
bladder, $\mathrm{P}=0.000061,5.7 \times 10^{-8}$ and $0.0099 . \mathrm{M}_{2}$ muscarinic receptor knockout mice, effects of isoproterenol: $E_{\max }$ in ileum and trachea, $\mathrm{P}=0.00093$ and $0.022 ; E C_{50}$ shift in ileum, trachea and urinary bladder, $\mathrm{P}=0.0021$, $9.7 \times 10^{-8}$ and 0.00022; $C E_{\text {observed }}$ in ileum, trachea and urinary bladder, $\mathrm{P}=0.000023,5.0 \times 10^{-8}$ and $3.4 \times 10^{-6}$.

Significantly different from the relaxant effect observed in wild type mice. The probability values for theses differences are as follows: Effects of isoproterenol on $E_{\max }$ in ileum, $\mathrm{P}=0.0089$; effects of isoproterenol on $E C_{50}$ shift in urinary bladder, $\mathrm{P}=0.0025$; effects of isoproterenol on $C E_{\text {observed }}$ in ileum and urinary bladder, $\mathrm{P}=$ 0.018 and 0.0059 . 
Table 4: Effect of pinacidil $(5.0 \mu \mathrm{M})$ on the contractile response to oxotremorine-M in smooth muscle from wild type and $\mathrm{M}_{2}$ muscarinic receptor knockout $(\mathrm{KO})$ mice. ${ }^{\text {a }}$

Ileum

Trachea

Urinary Bladder

Wild Type $(n=4)$
$125 \pm 11$
$3.2^{\mathrm{d}}$
$(0.50 \pm 0.059)$
$E_{\max }(\% \text { control })^{b}$
$80 \pm 4.1^{\mathrm{d}}$
$107 \pm 6.4$
$E C_{50}$ Shift $(\text { fold })^{c}$
$4.1^{\mathrm{d}}$
1.7
$(0.62 \pm 0.084)$
$(0.22 \pm 0.070)$

$\underline{M}_{2}$ Receptor KO$(n=4)$
$137 \pm 3.8^{\mathrm{d}}$
$2.2^{\mathrm{d}}$
$(0.35 \pm 0.055)$
$E_{\max }(\% \text { control })^{b}$
$E C_{50}$ Shift (fold) ${ }^{c}$
$87 \pm 4.4$
$111 \pm 9.5$
$3.9^{\mathrm{d}}$
$(0.59 \pm 0.070)$
1.5
$(0.19 \pm 0.070)$

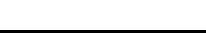

a Mean values \pm SEM from four wild type and four $\mathrm{M}_{2}$ receptor knockout mice are shown.

b Denotes the $E_{\max }$ value of oxotremorine-M measured in the presence of pinacidil divided by that measured in its absence, expressed as a percent.

c Denotes the $E C_{50}$ value of oxotremorine-M measured in the presence of pinacidil divided by that measured in its absence. The values in parentheses beneath each estimate represent the mean Log shift values \pm SEM.

d A significant effect of pinacicil relative to control. The probability values for these differences are as follows.

Wild type mice, effects of pinacidil on $E_{\max }$ in trachea, $\mathrm{P}=0.016 ; E C_{50}$ shift in ileum and trachea, $\mathrm{P}=0.0035$ and 0.0052. $\mathrm{M}_{2}$ muscarinic receptor knockout mice, effects of pinacidil on $E_{\max }$ in ileum, $\mathrm{P}=0.0022 ; E C_{50}$ shift in ileum and trachea, $\mathrm{P}=0.0079$ and 0.0035 . 


\section{$\underline{\text { Legends to Figures }}$}

Figure 1: Effects of forskolin on oxotremorine-M-mediated contractions in ileum $(A, D)$, trachea $(B, E)$ and urinary bladder $(C, F)$ from wild type $(A-C)$ and $M_{2}$ muscarinic receptor knockout mice (D-F). The contractile response to oxotremorine-M was measured in the absence $(\mathrm{O})$ and presence $(\bullet)$ of forskolin $(5.0 \mu \mathrm{M})$. The magnitudes of the contractions are expressed relative to the contraction elicited by $\mathrm{KCl}(50 \mathrm{mM})$. The data represent the mean values \pm SEM from five wild type and five knockout mice.

Figure 2: Effects of isoproterenol on oxotremorine-M-mediated contractions in ileum $(A, D)$, trachea (B,E) and urinary bladder $(C, F)$ from wild type $(A-C)$ and $M_{2}$ muscarinic receptor knockout mice $(D-F)$. The contractile response to oxotremorine-M was measured in the absence $(\mathrm{O})$ and presence $(\bullet)$ of isoproterenol $(1.0 \mu \mathrm{M})$. The magnitudes of the contractions are expressed relative to the contraction elicited by $\mathrm{KCl}(50 \mathrm{mM})$. The data represent the mean values $\pm \mathrm{SEM}$ from five wild type and five knockout mice.

Figure 3: Effects of forskolin $(5.0 \mu \mathrm{M})(\mathrm{A})$ and isoproterenol $(1.0 \mu \mathrm{M})(\mathrm{B})$ on contractions elicited to $\mathrm{KCl}(50 \mathrm{mM})$ in ileum, trachea and urinary bladder from wild type and $M_{2}$ muscarinic receptor knockout mice. The contractions are expressed as a percentage of the contraction elicited to $\mathrm{KCl}$ in the absence of the relaxant agents. The data represent the mean values + SEM from four wild type and four knockout mice. *, significantly different from the relaxant effect observed in wild type mice, $\mathrm{P}=0.039$. 

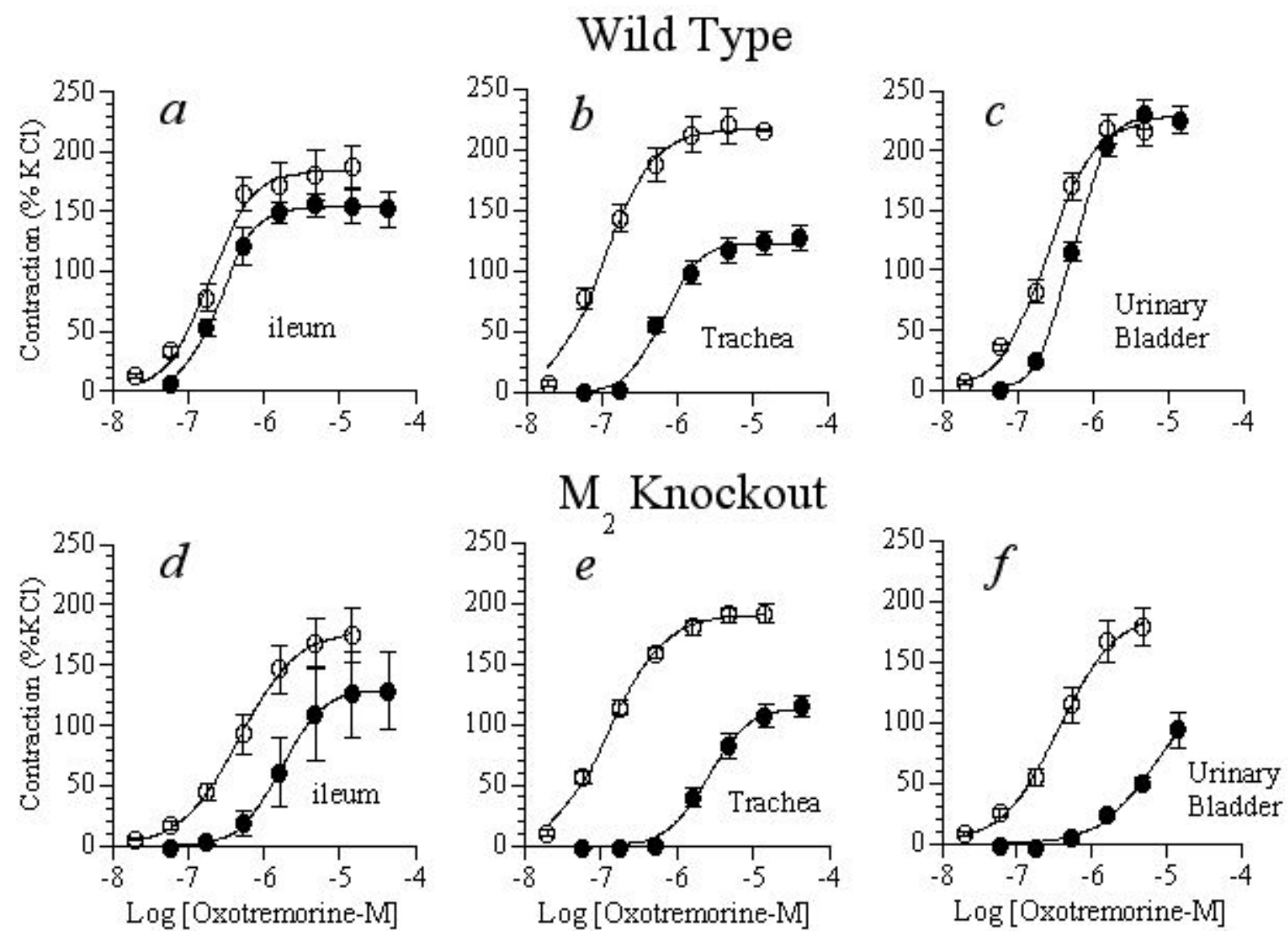

Figure 1 


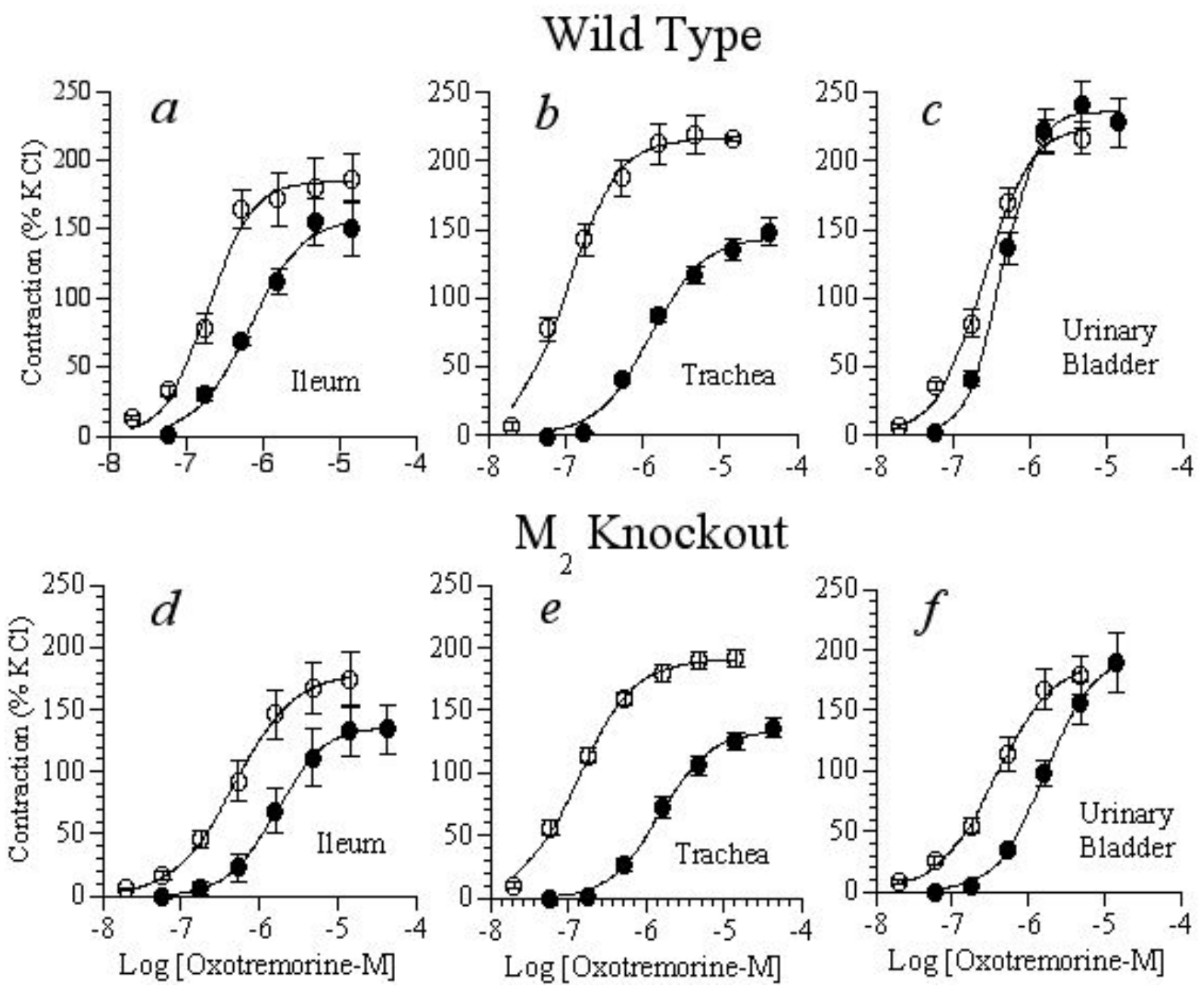

Figure 2 

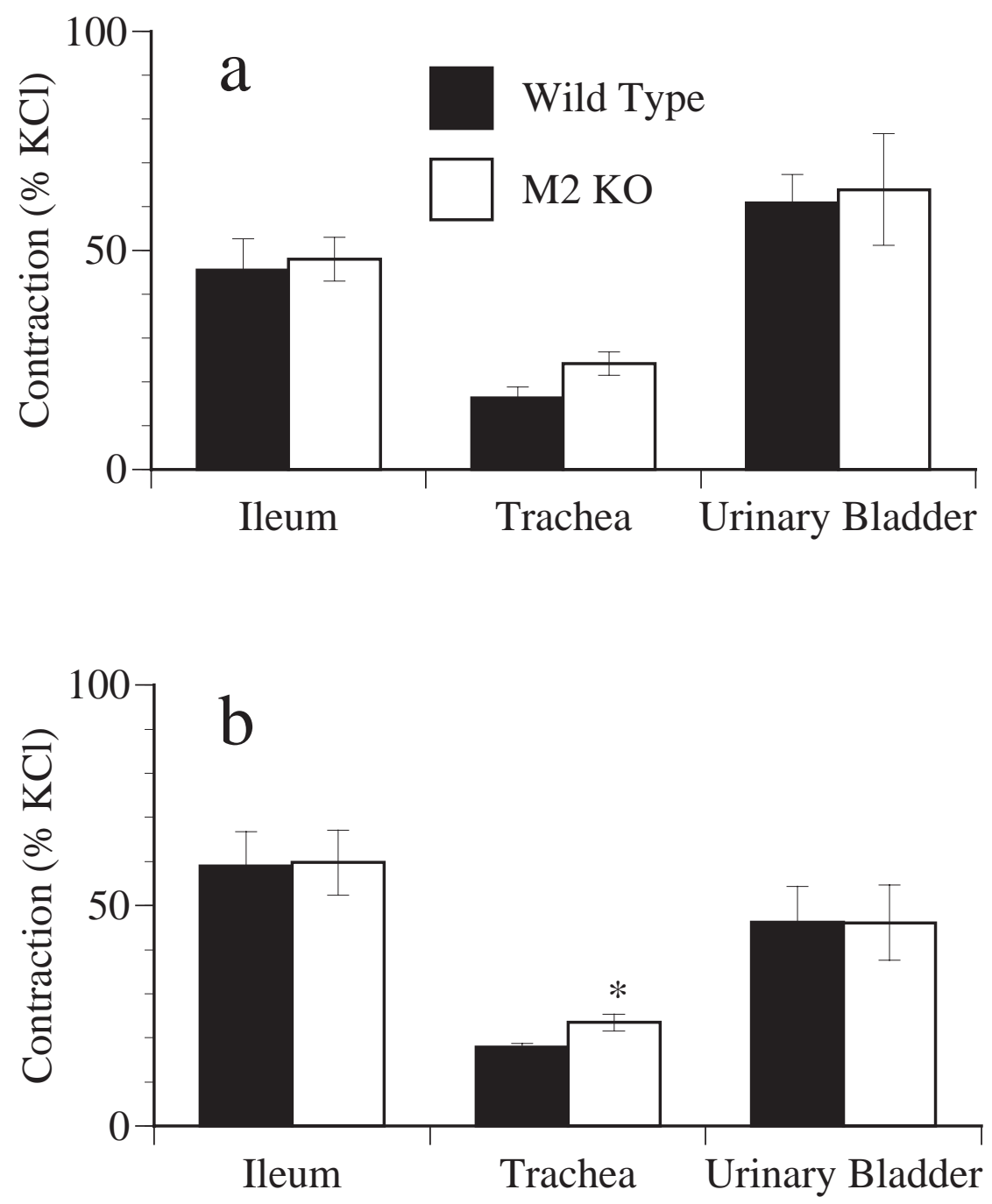

Figure 3 